\title{
Constructing a Low Energy Transfer Between Jovian Moons
}

\author{
Wang Sang Koon \\ Control and Dynamical Systems \\ Caltech 107-81, Pasadena, CA 91125 \\ koon@cds.caltech.edu
Jerrold E. Marsden
Control and Dynamical Systems
Caltech 107-81, Pasadena, CA 91125
marsden@cds.caltech.edu

\author{
Martin W. Lo \\ Navigation and Mission Design \\ Jet Propulsion Laboratory, Caltech \\ 4800 Oak Grove Drive, 301-142 \\ Pasadena, CA 91109-8099 \\ Martin.Lo@Jpl.Nasa.Gov \\ Shane D. Ross \\ Control and Dynamical Systems \\ Caltech 107-81, Pasadena, CA 91125 \\ shane@cds.caltech.edu
}

August 1, 2000

\begin{abstract}
There has recently been considerable interest in sending a spacecraft to orbit Europa, the smallest of the four Galilean moons of Jupiter. The trajectory design involved in effecting a capture by Europa presents formidable challenges to traditional conic analysis since the regimes of motion involved depend heavily on three-body dynamics. New three-body perspectives are required to design successful and efficient missions which take full advantage of the natural dynamics. Not only does a three-body approach provide low-fuel trajectories, but it also increases the flexibility and versatility of missions. We apply this approach to design a new mission concept wherein a spacecraft "leap-frogs" between moons, orbiting each for a desired duration in a temporary capture orbit. We call this concept the "Petit Grand Tour."

For this application, we apply dynamical systems techniques developed in a previous paper to design a Europa capture orbit. We show how it is possible, using a gravitional boost from Ganymede, to go from a jovicentric orbit beyond the orbit of Ganymede to a ballistic capture orbit around Europa. The main new technical result is the employment of dynamical channels in the phase space - tubes in the energy surface which naturally link the vicinity of Ganymede to the vicinity of Europa. The transfer $\Delta V$ necessary to jump from one moon to another is less than half that required by a standard Hohmann transfer.
\end{abstract}

\section{Introduction.}

Interest in Europa. Recently, there has been significant interest in sending a scientific spacecraft to orbit and study Europa, where possible subterranean oceans and life are suspected. One of the main difficulties with such a mission is the construction of a feasible trajectory from the Earth to Europa using a very limited fuel budget. One must take full advantage of the orbital dynamics in order to find even a reasonable first guess at such a trajectory. Fortunately, Jupiter's vicinity, and in particular the Galilean moons, provide enough interesting orbital dynamics that the construction of a relatively low fuel trajectory is possible.

In this study, we design a trajectory which ends in ballistic capture at Europa and beforehand executes a temporary capture around Ganymede. As this orbit resembles in miniature the Grand Tour of the solar 
system performed by the Voyager spacecraft, we call it a "Petit Grand Tour." One important difference between this Petit Grand Tour and the Voyager flybys is the "leap-frogging" strategy. In this novel approach to space mission design, the spacecraft can circle a moon in a loose temporary capture orbit for a desired number of orbits, perform a transfer $\Delta V$ and become ballistically captured by another adjacent moon for some number of orbits, etc. Instead of flybys lasting only seconds, a scientific spacecraft can orbit several different moons for any desired duration.

Taking Full Advantage of $N$-Body Dynamics. Taking full advantage of the $N$-body dynamics of a system, the fuel necessary to transfer between moons can be significantly less than that required by a Hohmann transfer. This is essentially due to the brute force nature of the Hohmann transfer which approximates each leg of the trajectory locally by 2-body solutions. Our approach patches 3-body solutions, which is particularly important for the ballistic capture regime of motion. In our study of a transfer from Ganymede to Europa, our transfer $\Delta V$ is half the Hohmann transfer value. We generate a tour of the Jovian moons: starting beyond Ganymede's orbit, the spacecraft is ballistically captured by Ganymede, orbits it once and escapes, and ends in a ballistic capture at Europa. See Figure 1.1.

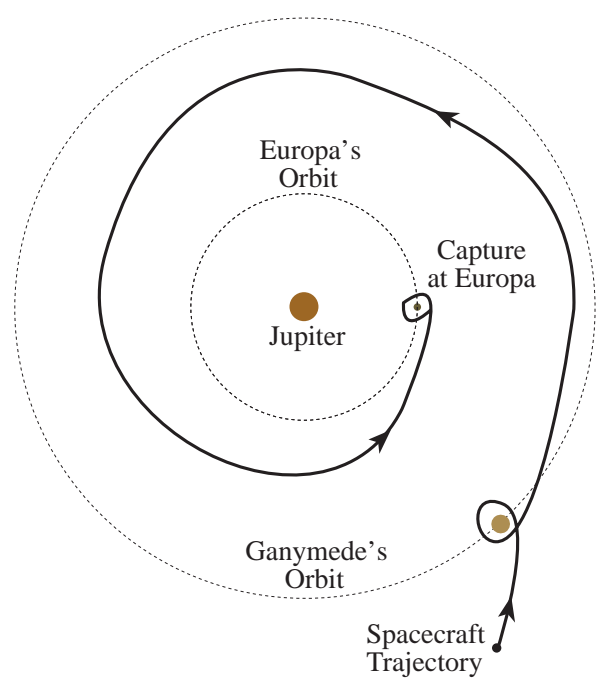

Figure 1.1: Tour of Jovian moons Ganymede and Europa (schematic).

Coupled Three-Body Model. Our nominal model for studying the natural dynamics governing transport between adjacent Galilean moons is the coupled 3-body problem. The coupled 3-body problem considers two nested 3-body systems, such as for two adjacent moons competing for control of the same spacecraft (e.g., Jupiter-Ganymede-spacecraft and Jupiter-Europa-spacecraft). When close to the orbit of one of the moons, the spacecraft's motion is dominated by the 3-body dynamics of the corresponding planet-moon system. Between the two moons, the spacecraft's motion is mostly planet-centered Keplerian, but is precariously poised between two competing 3-body dynamics. In this region, heteroclinic orbits connecting unstable Lagrange point orbits of the two different 3-body systems may exist, leading to complicated transfer dynamics between the two adjacent moons. Specifically, we will seek intersections between codimension-1 surfaces in the energy surface (dynamical channels or tubes) which connect the capture regions around each moon. The tubes in the energy surface separate transit orbits (inside the tube) from non-transit orbits (outside the tube). We will review the methodology of Koon, Lo, Marsden, and Ross [2000a] for finding such orbits below.

We study the 4-body problem invloving Jupiter (the massive central body), its two moons Europa and Ganymede (significantly less massive orbiting bodies), and the spacecraft (an effectively massless 
body which does not affect the motion of the others). The full 4-body problem is difficult to tackle in its entirety. Therefore we partition the problem into two 3-body problems, each involving Jupiter, a moon, and the spacecraft: the Jupiter-Europa-spacecraft system and the Jupiter-Ganymede-spacecraft system.

The simplest 3-body model we can consider which captures the essence of the natural dynamics is the planar circular restricted 3-body problem (PCR3BP). See Figure 1.2(a). The coupled PCR3BP considers two nested co-planar 3-body systems. In our case, we have a massive central planet with two moons on co-planar concentric circular orbits, and a spacecraft whose motion is also restricted to the common orbital plane. See Figure 1.2(b).

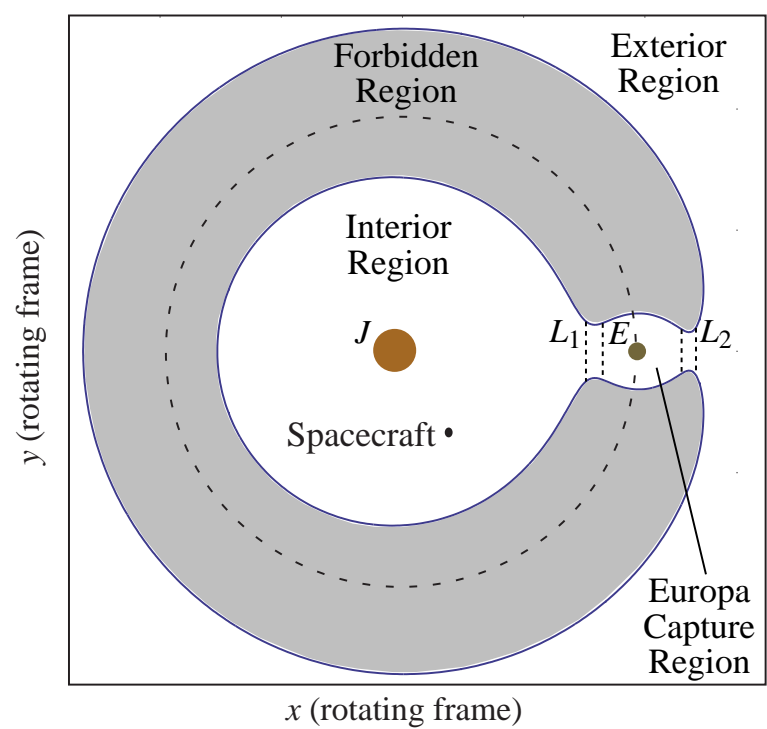

(a)

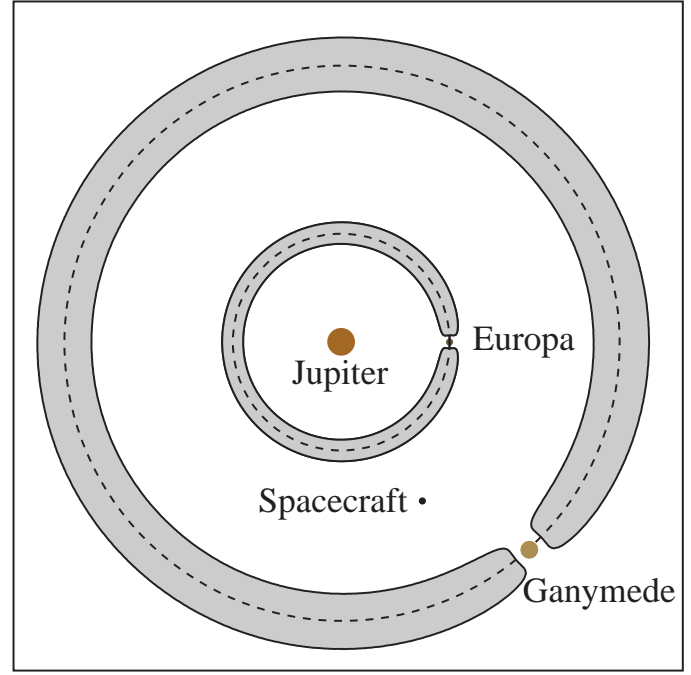

(b)

Figure 1.2: (a) PCR3BP model for Jupiter-Europa-spacecraft system; shown in frame co-rotating with Europa in its orbit. We work with 3-body energy regimes where region surrounding the moon's orbit (shaded) is energetically forbidden to spacecraft motion. Note small opening near moon, permitting capture. (b) Coupled PCR3BP: Four-body system approximated as two nested 3-body systems (schematic).

We use the coupled 3-body model to find an initial guess which we refine to obtain a trajectory in the 4-body bi-circular model. In the 4-body bi-circular model, both Ganymede and Europa are assumed to move in circular orbits about Jupiter in a common orbital plane. The motion of the spacecraft is also restricted to this plane. The initial guess trajectory is very close to the final integrated trajectory in the 4-body model. The fuel necessary for the transfer between the moons is less than half that required by a traditional Hohmman transfer.

\section{A Few Key Features of the Three-Body Problem.}

The Planar Circular Restricted Three-Body Problem. The orbital planes of Ganymede and Europa are within $0.3^{\circ}$ of each other, and their orbital eccentricities are 0.0006 and 0.0101 , respectively. If we restrict the motion of the spacecraft to their approximately common orbital plane, then the coupled PCR3BP is an excellent starting model for illuminating the transfer dynamics between these moons.

We begin by recalling the equations for the PCR3BP. See, for example, Szebehely [1967], Abraham and Marsden [1978], or Meyer and Hall [1992] for more information. The two main bodies, which we call generically Jupiter and the moon, have a total mass that is normalized to one. Their masses are denoted, as usual, by $m_{J}=1-\mu$ and $m_{M}=\mu$ respectively (see Figure 2.1(a)). These bodies rotate in the 


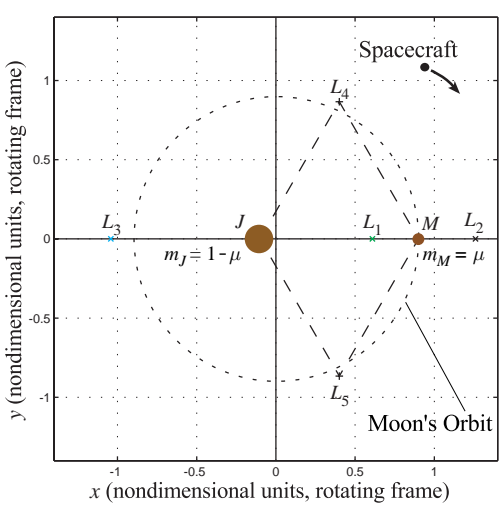

(a)

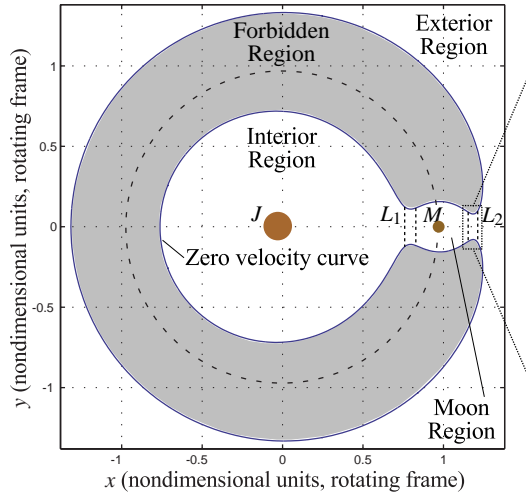

(b)

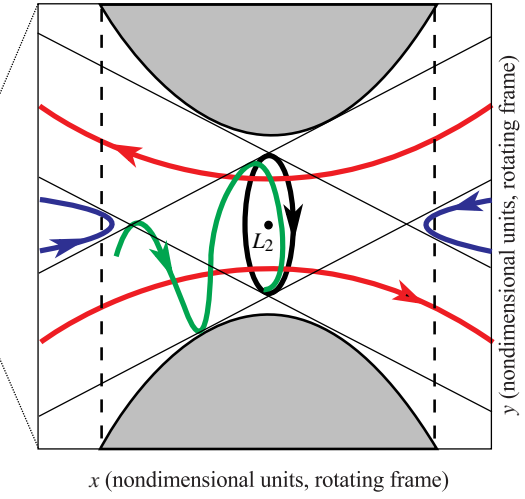

(c)

Figure 2.1: (a) Equilibrium points of the PCR3BP as viewed, not in any inertial frame, but in the rotating frame, where the Sun and Jupiter are at fixed positions along the $x$-axis. (b) Hill's region (schematic, the region in white), which contains a "neck" about $L_{1}$ and $L_{2}$. (c) The flow in the region near $L_{2}$, showing a periodic orbit around $L_{2}$, a typical asymptotic orbit winding onto the periodic orbit, two transit orbits and two non-transit orbits. A similar figure holds for the region around $L_{1}$.

plane counterclockwise about their common center of mass and with the angular velocity normalized to one. The third body, which we call the spacecraft, has mass zero and is free to move in the plane. Note that the mass parameters for the Jupiter-Ganymede and Jupiter-Europa systems are $\mu_{G}=7.802 \times 10^{-5}$ and $\mu_{E}=2.523 \times 10^{-5}$, respectively.

Choose a rotating coordinate system so that the origin is at the center of mass and Jupiter $(J)$ and the moon $(M)$ are fixed on the $x$-axis at $(-\mu, 0)$ and $(1-\mu, 0)$ respectively (see Figure 2.1(a)). Let $(x, y)$ be the position of the spacecraft in the plane (so these are the position coordinates relative to the positions of Jupiter and the moon, not relative to an inertial frame).

Equations of Motion. There are several ways to derive the equations of motion for this system, as discussed at length in the above references. For example, as in Whittaker's book, Abraham and Marsden [1978] use time dependent canonical transformation theory to transform the problem from an inertial frame to a rotating frame. This method gives the equations in Hamiltonian form and the associated Lagrangian form is given by the Legendre transformation.

A simpler technique is to use covariance of the Lagrangian formulation and use the Lagrangian directly in a moving frame (see Marsden and Ratiu [1999]). This method directly gives the equations in Lagrangian form. Then the equations of motion of the spacecraft can be written in second order form as

$$
\ddot{x}-2 \dot{y}=\Omega_{x}, \quad \ddot{y}+2 \dot{x}=\Omega_{y}
$$

where

$$
\Omega=\frac{x^{2}+y^{2}}{2}+\frac{1-\mu}{r_{1}}+\frac{\mu}{r_{2}}+\frac{\mu(1-\mu)}{2},
$$

and where $\Omega_{x}, \Omega_{y}$ are the partial derivatives of $\Omega$ with respect to the variables $x, y$. Here, $r_{1}$ and $r_{2}$ are the distances of the spacecraft from the two main bodies and are given by $r_{1}=\sqrt{(x+\mu)^{2}+y^{2}}$ and $r_{2}=\sqrt{(x-1+\mu)^{2}+y^{2}}$.

This system of equations are called the equations of the PCR3BP and have been studied in detail in Szebehely [1967]. They have a first integral called the Jacobi integral, which is given by

$$
C(x, y, \dot{x}, \dot{y})=-\left(\dot{x}^{2}+\dot{y}^{2}\right)+2 \Omega(x, y) .
$$


Equilibrium Points and Hill's Regions. The system (2.1) has five equilibrium points, 3 collinear ones on the $x$-axis, called $L_{1}, L_{2}, L_{3}$ and two equilateral points called $L_{4}, L_{5}$ (see Figure 2.1(a)). These equilibrium points are critical points of the (effective potential) function $\Omega$. The value of the Jacobi integral at the point $L_{i}$ will be denoted by $C_{i}$.

The level surfaces of the Jacobi constant, which are also energy surfaces, are invariant 3-dimensional manifolds. Let $\mathcal{M}$ be that energy surface, i.e.,

$$
\mathcal{M}(\mu, C)=\{(x, y, \dot{x}, \dot{y}) \mid C(x, y, \dot{x}, \dot{y})=\mathrm{constant}\}
$$

The projection of this surface onto position space is called a Hill's region

$$
M(\mu, C)=\{(x, y) \mid \Omega(x, y) \geq C / 2\} .
$$

The boundary of $M(\mu, C)$ is the zero velocity curve. The spacecraft can move only within this region in the $(x, y)$-plane. Our main concern here is the behavior of the orbits of equations (2.1) whose Jacobi constant is just below that of $L_{2}$, that is, $C<C_{2}$. For this case, the Hill's region contains a "neck" about $L_{1}$ and $L_{2}$, as shown in Figure 2.1(a). Thus, orbits with a Jacobi constant just below that of $L_{2}$ are energetically permitted to make a transit through the two neck regions from the interior region (inside the moon's orbit) to the exterior region (outside the moon's orbit) passing through the moon (capture) region.

The Flow near the Lagrange Points $L_{1}$ and $L_{2}$. Having fixed on an appropriate energy level surface, we first study the behavior of orbits near the equilibrium points (see Figure 2.1(c)). Conley [1963, 1968] performed a local study using the linearized system of the PCR3BP. With the aid of a theorem of Moser, all the qualitative results of this linearized system carry over to the full nonlinear equations.

The flows near $L_{1}$ and $L_{2}$ are similar. The neck around each equilibrium point is referred to as the equilibrium region. To summarize Conley's results, in each equilibrium region around $L_{1}$ or $L_{2}$, there exist four types of orbits (see Figure 2.1(c)): the periodic Lyapunov orbit, the cylindrical sets of asymptotic orbits that wind onto this periodic orbit (pieces of the periodic orbit's stable and unstable invariant manifold), the transit orbits that the spacecraft uses to make a transit from one region to the other and, finally, the non-transit orbits where the spacecraft comes out of one region and pass near the equilibrium point only to fall back into the same region.

The key observation here, first made by McGehee [1969], is that the asymptotic orbits are pieces of the 2-dimensional stable and unstable invariant manifold "tubes" associated to the Lyapunov orbit and they separate two distinct types of motion in the 3-dimensional energy surface: transit orbits and non-transit orbits. The transit orbits, passing from one region to another, are those inside the cylindrical manifold tube. The non-transit orbits, which bounce back to their region of origin, are those outside the tube. Most importantly for our purposes, to transit from outside a moon's orbit to inside (or vice versa), or get temporarily captured, one must be inside the tubes of transit orbits. See Figure 2.2.

We emphasize that a trajectory must be inside these stable and unstable manifold tubes to transit from one side of the Lyapunov orbit to the other, meaning from one region to another (e.g., exterior to Ganymede capture region in Figure 2.2(a)). If the unstable manifold of the $L_{i}$ Lyapunov orbit intersects the stable manifold of the $L_{3-i}$ Lyapunov orbit, then there exist transit orbits going from the interior (resp. exterior) region to the exterior (resp. interior) region, where $i=1$ (resp. 2). If the trajectory in the moon capture region is in a portion of the tube which wraps around the moon, we call it temporarily captured (Figure 2.2(b)). A small $\Delta V$ would change the Jacobi constant of a spacecraft to one in which the necks at $L_{1}$ and $L_{2}$ have sealed off $\left(C>C_{1}\right)$, resulting in permanent capture.

Heteroclinic Connections. A numerical demonstration is given in Koon, Lo, Marsden, and Ross [2000a] of a heteroclinic connection between pairs of equal Jacobi constant Lyapunov orbits, one around $L_{1}$, the other around $L_{2}$. This heteroclinic connection augments the homoclinic orbits associated with the $L_{1}$ and $L_{2}$ Lyapunov orbits, which were previously known (McGehee [1969]). Linking these heteroclinic connections and homoclinic orbits leads to dynamical chains. 


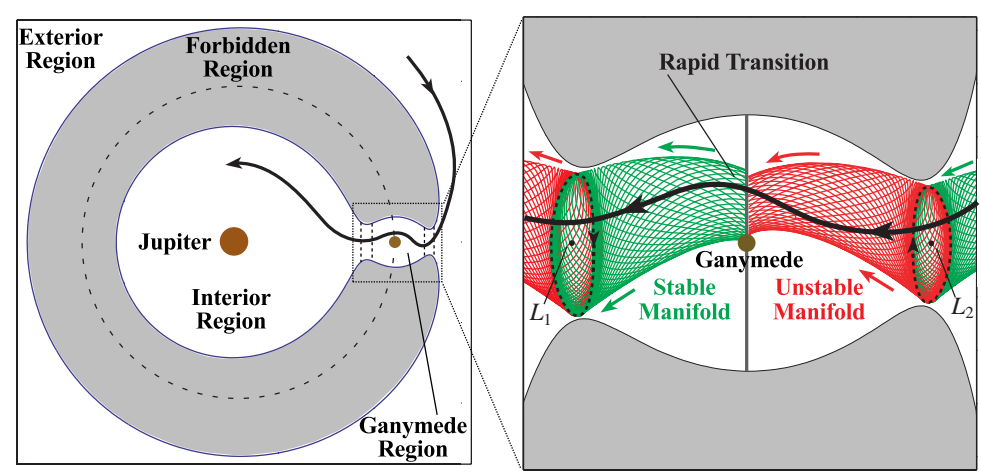

(a)

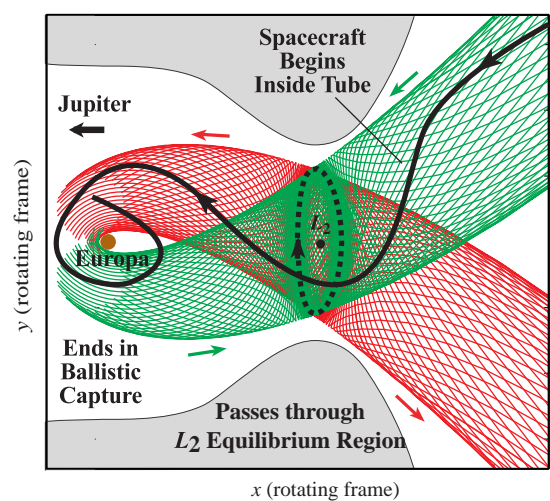

(b)

Figure 2.2: (a) Transit orbit from outside to inside Ganymede's orbit, passing by Ganymede. The tubes of transit orbits (bounded by the cylindrical stable (lightly shaded) and unstable (darkly shaded) manifolds) intersect such that a transition is possible. (b) Orbit that ends in ballistic capture at Europa.

Existence and Numerical Construction of Orbits with Prescribed Itineraries. Koon, Lo, Marsden, and Ross [2000a] proved the existence of a large class of interesting orbits near a chain which a spacecraft can follow in its rapid transition between the inside and outside of a Jovian moon's orbit via a moon encounter. The global collection of these orbits is called a dynamical channel. We shall also use this term when collections of such chains for separate 3-body systems, roughly speaking, overlap and are put end to end. We can individually label the orbits near a chain with an itinerary giving their past and future whereabouts, making their classification and manipulation possible. Koon, Lo, Marsden, and Ross [2000a] not only proved the existence of orbits with prescribed itineraries, but develop a systematic procedure for their numerical construction. This is an important part of the program; it turns a general existence theory into a practical technique for constructing orbits.

Koon, Lo, Marsden, and Ross [2000a] used chains of homoclinic and heteroclinic orbits to construct a suitable Poincaré map which allows one to classify as well as organize distinctively different types of global motions of the PCR3BP in terms of ultimate behavior with respect to the equilibrium points. They proved a theorem which gives the global orbit structure in the neighborhood of a chain. In simplified form, the theorem essentially says:

For any admissible bi-infinite sequence $\left(\ldots, u_{-1} ; u_{0}, u_{1}, u_{2}, \ldots\right)$ of symbols $\{I, M, X\}$ where $I, M$, and $X$ stand for the interior, moon, and exterior regions respectively, there corresponds an orbit near the chain whose past and future whereabouts with respect to these three regions match those of the given sequence.

For example, consider the Jupiter-Ganymede-spacecraft 3-body system. Given the bi-infinite sequence $(\ldots, I ; M, X, M, \ldots)$, there exists an orbit starting in the Ganymede region which came from the interior region and is going to the exterior region and returning to the Ganymede region.

The set of orbits which remain eternally in a small neighborhood of a chain, which we refer to as the invariant set, is a theoretical construct, and though useful for guiding our understanding and classification of the dynamics, its infinite nature renders it powerless to provide us with usable trajectories. However, by using the proof of the theorem as a guide, computational and numerical methods can be brought to bear which iteratively approximate the invariant set and allow us to develop a systematic procedure to numerically construct orbits with finite prescribed itineraries.

The description of the construction of the invariant set in Koon, Lo, Marsden, and Ross [2000a] involved successive iterations of the Poincaré map $P$. Finite areas of finite central block itineraries evolved under successive application of the map $P$ into a "cloud of points," the invariant set $\Lambda$ of points 
with bi-infinite itineraries. If we truncate the construction of the invariant set at some finite number of iterations of $P$, we will find regions of phase space which have a certain finite itinerary. The essential feature of the orbit, its itinerary, will be robust because all the nearby orbits in phase space have the same finite itinerary. Thus, by truncating our construction of the invariant set $\Lambda$ at some finite number of applications of $P$, we can generate a set of robust orbits with different finite itineraries. The sets of orbits with different itineraries are easily visualizable on our chosen Poincaré section as areas in which all the orbits have the same finite itinerary. We will also no longer be limited to a small neighborhood of a chain, but can obtain more global results.

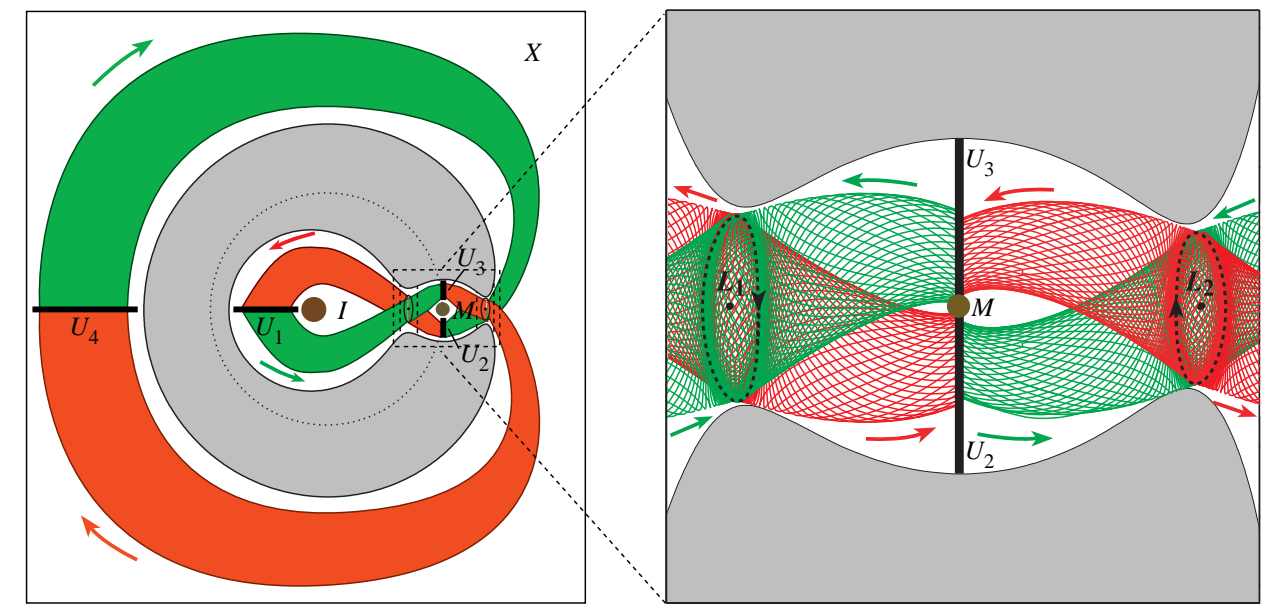

Figure 2.3: Location of Lagrange point orbit invariant manifold tubes in position space. Stable manifolds are lightly shaded, unstable manifolds are darkly shaded. The location of the Poincaré sections $\left(U_{1}, U_{2}, U_{3}\right.$, and $\left.U_{4}\right)$ are also shown.

Example Itinerary: $(M, X ; M, I, M)$. We illustrate the numerical construction of orbits with prescribed finite (but arbitrarily large) itineraries in the three-body planet-moon-spacecraft problem. As our example, chosen for simplicity of exposition, we construct a spacecraft orbit with the central block $(M, X ; M, I, M)$.

For the present numerical construction, we adopt the following convention. The $U_{1}$ and $U_{4}$ Poincaré sections will be the planes $(y=0, x<0)$ in the interior region, and $(y=0, x<-1)$ in the exterior region, respectively. The $U_{2}$ and $U_{3}$ sections will be the planes $(x=1-\mu, y<0)$ and $(x=1-\mu, y>0)$ in the moon region, respectively. See Figure 2.3 for the location of the Poincaré sections relative to the tubes. A key observation here is a result of McGehee [1969] which has shown that the invariant manifold tubes separate two types of motion. See Figures 2.4(a) and 2.4(b). The orbits inside the tube transit from one region to another; those outside the tubes bounce back to their original region.

Since the upper curve in Figure 2.4(b) is the Poincaré cut of the stable manifold of the periodic orbit around $L_{1}$ in the $U_{3}$ plane, a point inside that curve is an orbit that goes from the moon region to the interior region, so this region can be described by the label $(; M, I)$. Similarly, a point inside the lower curve of Figure 2.4(b) came from the exterior region into the moon region, and so has the label $(X ; M)$. A point inside the intersection $\Delta_{\mathcal{M}}$ of both curves is an $(X ; M, I)$ orbit, so it makes a transition from the exterior region to the interior region, passing through the moon region. Similarly, by choosing Poincaré sections in the interior and the exterior region, i.e., in the $U_{1}$ and $U_{4}$ plane, we find the intersection region $\Delta_{\mathcal{I}}$ consisting of $(M ; I, M)$ orbits, and $\Delta_{\mathcal{X}}$, which consists of $(M ; X, M)$ orbits.

Flowing the intersection $\Delta_{\mathcal{X}}$ forward to the moon region, it stretches into the strips in Figure 2.4(c). These strips are the image of $\Delta_{\mathcal{X}}$ (i.e., $P\left(\Delta_{\mathcal{X}}\right)$ ) under the Poincaré map $P$, and thus get the label $(M, X ; M)$. Similarly, flowing the intersection $\Delta_{I}$ backward to the moon region, it stretches into the strips $P^{-1}\left(\Delta_{\mathcal{I}}\right)$ in Figure 2.4(c), and thus have the label $(; M, I, M)$. The intersection of these two types 

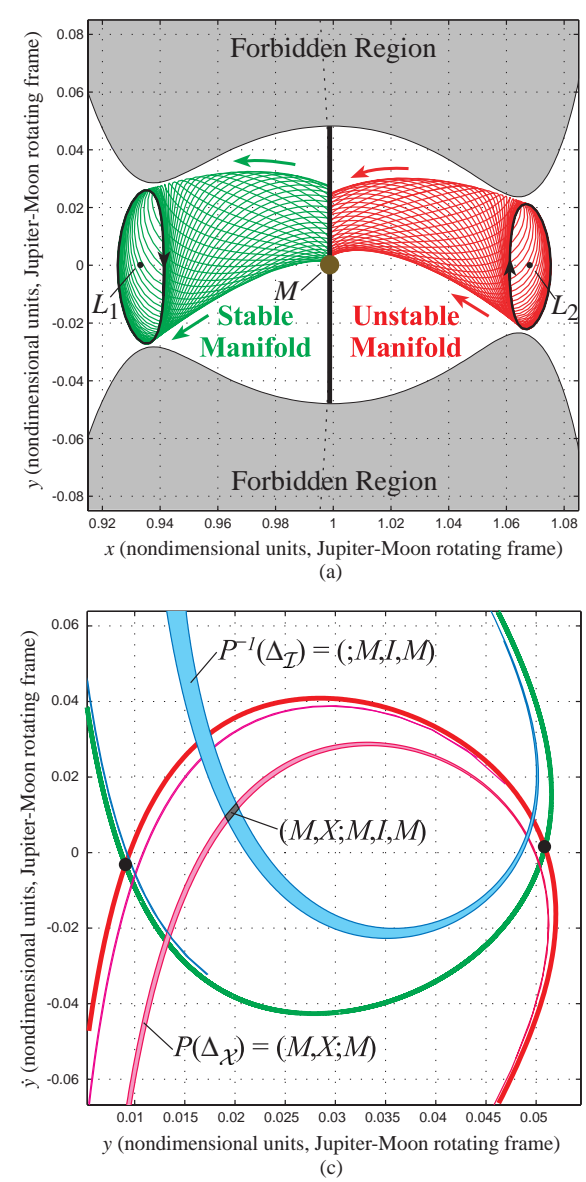

(c)

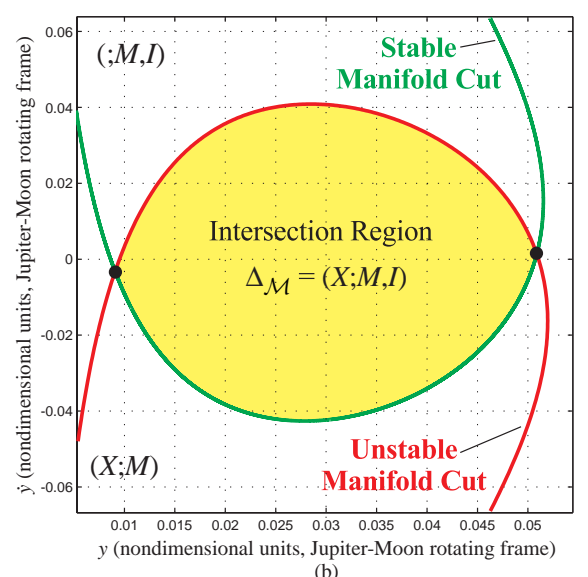

(b)

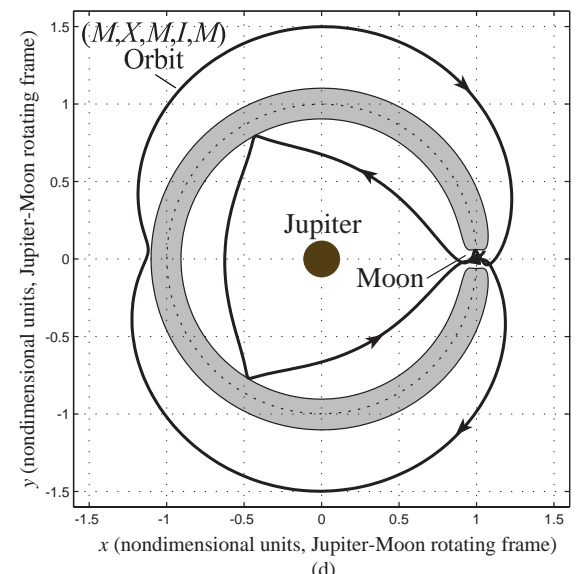

(d)

Figure 2.4: (a) The projection of invariant manifolds $W_{L_{1}, \text { p.o. }}^{s, \mathcal{M}}$ and $W_{L_{2} \text {,p.o. }}^{u, \mathcal{M}}$ in the region $M$ of the position space. (b) A close-up of the intersection region between the Poincaré cuts of the invariant manifolds on the $U_{3}$ section $(x=1-\mu, y>0)$. (c) Intersection between image of $\Delta_{X}$ and pre-image of $\Delta_{I}$ labeled $(M, X ; M, I, M)$. (d) Example orbit passing through $(M, X ; M, I, M)$ region of (c).

of strips (i.e., $\Delta_{\mathcal{M}} \cap P\left(\Delta_{\mathcal{X}}\right) \cap P^{-1}\left(\Delta_{\mathcal{I}}\right)$ ) consist of the desired $(M, X ; M, I, M)$ orbits. If we take any point inside these intersections and integrate it forward and backward, we find the desired orbits. See Figure 2.4(d).

\section{Ganymede to Europa Transfer with Ballistic Capture.}

Petit Grand Tour. The techniques developed thus far, especially the construction of orbits with prescribed itineraries, have been used to produce the Petit Grand Tour of Jovian moons.

Our approach to effect a Ganymede to Europa transfer with a ballistic ("free") capture at Europa builds on the theoretical understanding developed in Koon, Lo, Marsden, and Ross [2000a] as well as the following three key ideas: (1) treat the Jupiter-Ganymede-Europa-spacecraft 4-body problem as two coupled circular restricted 3-body problems, Jupiter-Ganymede-spacecraft and Jupiter-Europaspacecraft systems, (2) use the stable and unstable manifolds of periodic Lyapunov orbits about the Jupiter-Ganymede Lagrange points to provide a low energy transfer from beyond the orbit of Ganymede to a temporory capture around Ganymede, to the stable manifolds of periodic orbits around the JupiterEuropa Lagrange points, (3) use the stable manifolds of the periodic orbits around the Jupiter-Europa 
Lagrange points to provide a ballistic capture about Europa. The construction is done mainly in the Jupiter-Europa rotating frame using a Poincaré section which helps to glue the Jupiter-Ganymede libration point trajectory with the Europa ballistic capture trajectory.

We start with the PCR3BP model to compute the invariant manifolds and use them to construct the Jupiter-Ganymede libration point trajectory and the Europa ballistic capture trajectory. The final trajectory starting from beyond Ganymede's orbit and ending in Europa capture is integrated in the bicircular 4-body model where both Ganymede and Europa are assumed to move in circular orbits about Jupiter, and the spacecraft is an infinitesimal mass point. All the bodies move in a common orbital plane.

Ganymede to Europa Transfer Mechanism. We begin our construction from the transfer patch point and work forward and backward in time toward each moon's vicinity. To effect the Ganymede to Europa transfer, we compute Jupiter-Ganymede invariant unstable manifolds of an $L_{1}$ periodic orbit (interior region) and Jupiter-Europa invariant stable manifolds of an $L_{2}$ periodic orbit (exterior region). We seek an orbit which passes from Ganymede's vicinity to inside Ganymede's orbit. This region is outside Europa's orbit, thus we want the orbit to subsequently go from outside Europa's orbit to Europa's vicinity. See Figure 3.1.

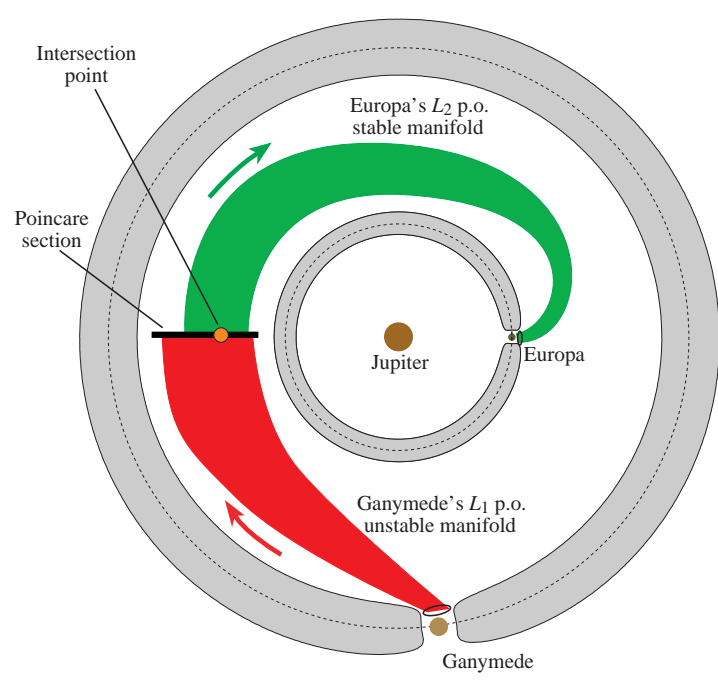

(a)

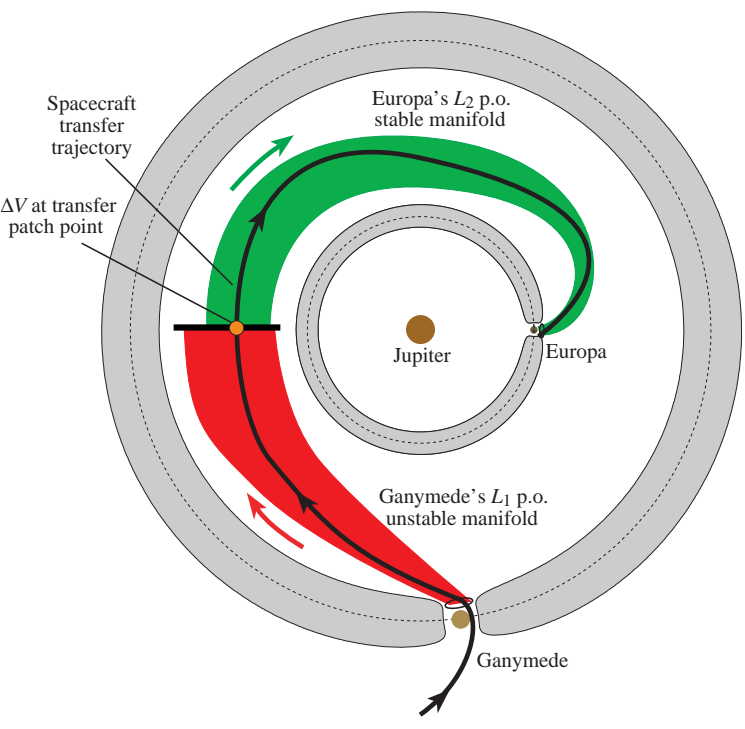

(b)

Figure 3.1: (a) Find an intersection between dynamical channel enclosed by Ganymede's $L_{1}$ periodic orbit unstable manifold and dynamical channel enclosed by Europa's $L_{2}$ periodic orbit stable manifold (shown in schematic). (b) Integrate forward and backward from patch point (with $\Delta V$ to take into account velocity discontinuity) to generate desired transfer between the moons (schematic).

We look at the intersection of these manifolds on a common Poinaré section. In particular, the section we choose is the $U_{4}$ section of the Jupiter-Europa rotating frame (see Figure 2.3). This fixes the phase of Europa (with respect to the patch point) at the time of the transfer maneuver. For simplicity, we choose the $U_{1}$ section of the Ganymede $L_{1}$ manifold of the Jupiter-Ganymede rotating frame and choose the phase of Ganymede such that this plane coincides with the $U_{4}$ section of the Jupiter-Europa rotating frame at the time of the transfer maneuver. Since the intersections of the manifold tubes are closed curves, the transformation of the Ganymede $L_{1}$ manifold from the Jupiter-Ganymede rotating frame to the Jupiter-Europa rotating frame is also a closed curve. Note that the region enclosed by these manifolds in the energy surface of each system respectively is the dynamical channel of the respective system.

The size of the channel in the phase space and on the Poincaré section is determined by the size 
(amplitude) of the corresponding Lyapunov orbit and therefore the size of the equilibrium region neck. We want to choose the "skinniest" possible Europa channel, corresponding to a skinny Europa $L_{2}$ neck. This is the Europa energy range where a capture is barely possible ( $C$ just slightly less than $C_{2}$ ) and where a natural temporary capture trajectory will perform the most circuits around Europa before escaping. The Europa $L_{2}$ stable channel (i.e., stable manifold tube), call it $\mathcal{C}_{E}^{s}$, has a particular Jacobi constant value $C_{E}$, as computed in the Jupiter-Europa rotating frame.

In order to have a Ganymede $L_{1}$ unstable channel $\mathcal{C}_{G}^{u}$ which intersects or closely approaches the Europa $L_{2}$ channel in the phase space, we must choose a large Ganymede $L_{1}$ Lyapunov orbit. We expect that there is some minimum size Ganymede $L_{1}$ Lyapunov orbit at which phase space intersections occur. But we do not want the Ganymede $L_{1}$ Lyapunov orbit too large because this implies an energy regime where revolutions around Ganymede are not possible (the orbit simply passes by Ganymede). We would like, if possible, to include at least one circuit around Ganymede.

Numerical Computation of Channel Intersections. For our numerical study we choose a Europa $L_{2}$ Lyapunov orbit with Jupiter-Europa Jacobi constant $C_{E}=3.0028$ (amplitude 0.26) and a Ganymede $L_{1}$ Lyapunov orbit with Jupiter-Ganymede Jacobi constant $C_{G}=3.0576$ (amplitude 0.35). The amplitudes roughly correspond to half the $x$-width of the Lyapunov orbit and are measured in units of the libration point to moon distance. These particular Jacobi constants (and the amplitudes they fix) are chosen because they are known, from numerical experiments, to lead to a dynamical channel intersection in position space.

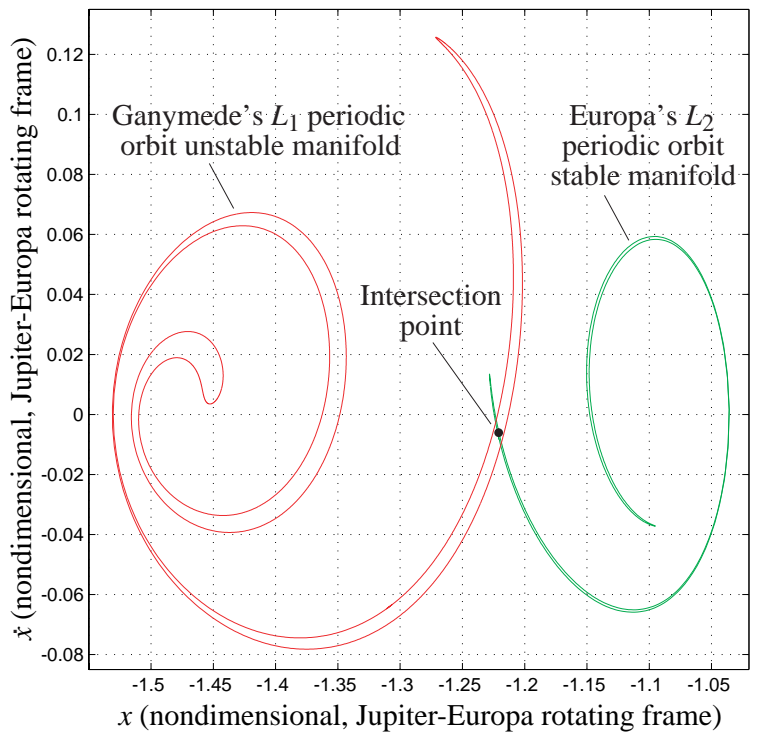

(a)

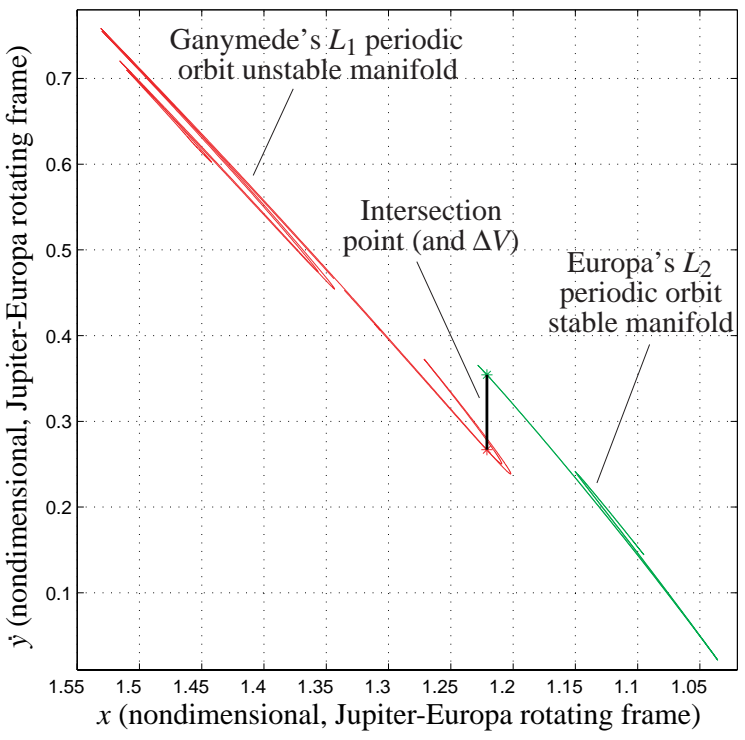

(b)

Figure 3.2: Poincaré section where intersection point for transfer found: (a) $(x, \dot{x})$-plane, (b) $(x, \dot{y})$-plane. Note that the manifolds in (b) lie on roughly constant lines, corresponding to constant Jacobi integral in each system respectively.

In each system respectively, we compute the Lyapunov orbit manifolds. See Figure 3.2. For the Jupiter-Europa system, we compute the $L_{2}$ exterior stable manifold (dark curve in figures). For the Jupiter-Ganymede system, we compute the $L_{1}$ interior unstable manifold (light curve in figures). Again, in each rotating system respectively, we choose a Poincaré section of the manifold along a ray emanating from Jupiter at a certain longitudinal angle $\theta$ from the moon. We denote the Europa angle by $\theta_{E}$ and the Ganymede angle by $\theta_{G}$. The Poincaré section obtained in each system is in polar coordinates $(r, \theta=$ constant, $\dot{r}, r \dot{\theta})$ as measured separately in each system's rotating frame. As mentioned earlier, we have chosen for simplicity the $U_{1}$ plane in the Ganymede system and the $U_{4}$ plane in the Europa system. 
This corresponds to $\theta_{E}=\theta_{G}=\pi$, for which the polar coordinates of the Poincaré section become identical to the cartesian coordinates $(x, y=0, \dot{x}, \dot{y})$ for each rotating system, respectively.

We choose the Jupiter-Europa rotating frame as our preferred frame of reference. Thus, the Europa manifold, or the Europa channel, is time invariant. To look for intersections between the Europa and Ganymede channels, we will transform the Ganymede manifold Poincaré section into the Europa frame. This is simply a rescaling of the coordinates. Note that since Ganymede is seen to move in a circular retrograde orbit as seen in Europa's rotating frame (i.e., its phase angle $\theta_{G}^{\prime}$ is a periodic function of time), Ganymede's channel is time dependent (specifically, periodic).

Take the time of the Poincaré section to be $t=0$. We want the Poincaré section in the Europa frame to coincide with the same Poincare section in the Ganymede frame. We therefore choose Ganymede to have a phase at $t=0$ of $\theta_{G}^{\prime}=\theta_{E}-\theta_{G}$, as seen in the Europa frame. For this initial study, we choose $\theta_{E}=\theta_{G}=\pi$, for which $\theta_{G}^{\prime}=0$, i.e., Jupiter, Europa, Ganymede, and the spacecraft are all aligned on the $x$-axis as seen in the Europa rotating frame at $t=0$.

The Poincare section showing the cross-section of the Europa and Ganymede manifolds (and their interiors) in $(x, \dot{x})$ coordinates is shown in Figure 3.2(a). The region enclosed by the manifolds are the dynamical channels which connect the capture regions of each moon respectively. The area enclosed by the dark curve is the cross-section of the Ganymede $L_{1}$ dynamical channel and the area enclosed by the light curve is the cross-section of the Europa $L_{2}$ dynamical channel.

Notice the intersection around $(x=-1.22, \dot{x}=-0.005)$ in Figure 3.2(a). Ideally, this intersection implies that one can transit from Ganymede to Europa with no $\Delta V$ in the $x$-direction. Figure 3.2(b) shows the Poincaré section in $(x, \dot{y})$ coordinates, where for $x=-1.22$ we see a significant gap between the Ganymede and Europa channels. Therefore, a significant $\Delta V$ in the $y$-direction will be necessary. This is due to the fact that the Ganymede $L_{1}$ channel is not on the same Jupiter-Europa Jacobi constant surface as the Europa $L_{2}$ channel.

The Ganymede $L_{1}$ channel is on a Jupiter-Ganymede Jacobi constant surface, but spans a significant range of Jupiter-Europa Jacobi integrals, corresponding roughly to the "width" of the tilted rectangle which the cross-section occupies. The appearance of the Ganymede $L_{1}$ channel on the $(x, \dot{x})$-plane is a spiral (Figure 3.2(a)) and the Jupiter-Europa Jacobi integral of the channel decreases as one moves away from the center along the spiral. Therefore, the smallest values of the Jupiter-Europa Jacobi constant along the Ganymede $L_{1}$ channel are near the end of the dark curve spiral in Figure 3.2(a). The smallest value (3.0576) is significantly higher than the constant Jupiter-Europa Jacobi integral of the Europa $L_{2}$ channel (3.0028). From the difference in Jupiter-Europa Jacobi integral values, we can estimate, using the equation for the Jacobi integral (2.2), that a $\Delta V$ of about $1209 \mathrm{~m} / \mathrm{s}$ in the $y$-direction will be necessary to transition from the Ganymede $L_{1}$ channel to the Europa $L_{2}$ channel. This is the length of the vertical line connecting the two manifolds in Figure 3.2(b) (after scaling from nondimensional to SI units).

Four-Body System Approximated by Coupled PCR3BP. In order to determine an accurate $\Delta V$, we compute the transfer trajectory in the full 4-body system, taking into account the gravitational attraction of all three massive bodies on the spacecraft. We use the dynamical channel intersection region in the coupled 3-body model as an initial guess which we adjust finely to obtain a true 4-body bi-circular model trajectory. We expect a $\Delta V$ of about 1209 meters/second will be necessary to connect the two natural "capture" trajectories (i.e., forward capture by Europa and backward capture by Ganymede).

There are two initial conditions: one which backward integrates to Ganymede and one which forward integrates to Europa. These two have the same position, but differ in velocity. The initial velocity which integrates backward to Ganymede is chosen in the Ganymede dynamical channel. The initial velocity which integrates forward to Europa is chosen in the Europa dynamical channel. The difference in velocity is recorded as the $\Delta V$ necessary to transition from one natural trajectory to the other.

Figure 3.3 is the final end-to-end trajectory. A $\Delta V$ of $1208 \mathrm{~m} / \mathrm{s}$ is required at the location marked. This is remarkably close to the simple Jacobi integral approximation above, validating the coupled 3-body model as an excellent approximation to the 4-body dynamics of this system. We note that a traditional Hohmann (patched 2-body) transfer from Ganymede to Europa requires a $\Delta V$ of $2822 \mathrm{~m} / \mathrm{s}$. Our value 
is only $42.9 \%$ of the Hohmann value, which is a substantial savings of onboard fuel. The transfer flight time is about 25 days, well within conceivable mission constraints.

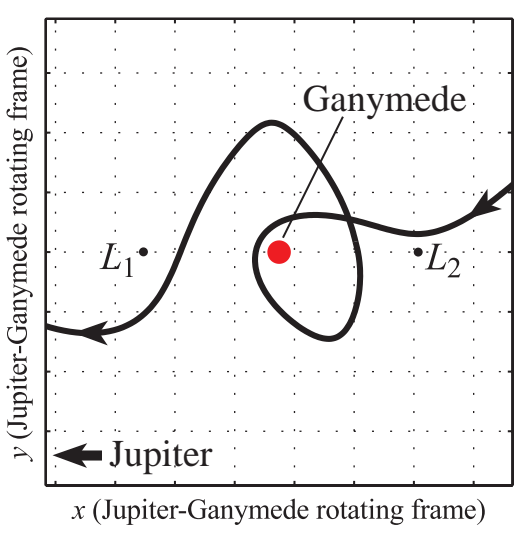

(a)

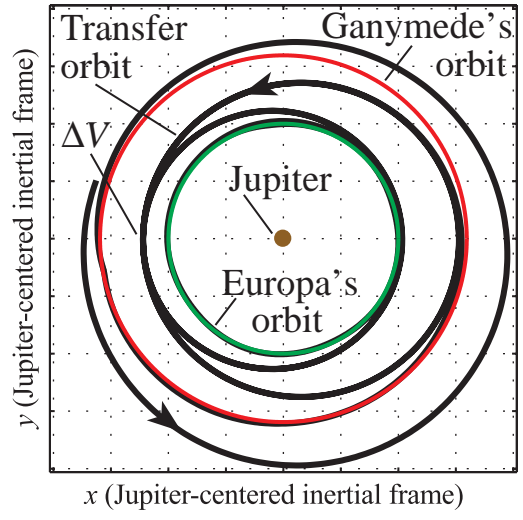

(b)

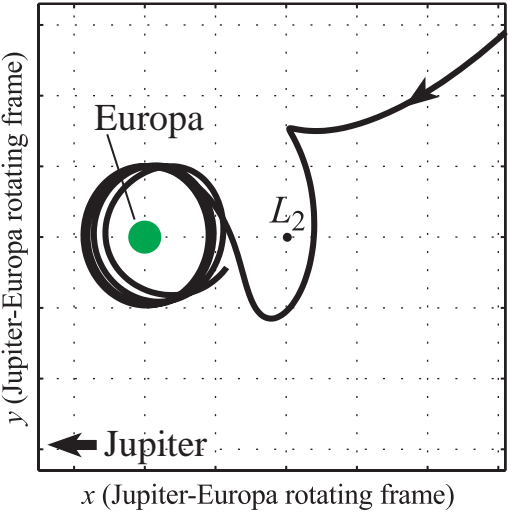

(c)

Figure 3.3: The "Petit Grand Tour" space mission concept for the Jovian moons. We show an orbit coming into the Jupiter system and (a) performing one loop around Ganymede (shown in the Jupiter-Ganymede rotating frame), (b) transferring from Ganymede to Europa using a single impulsive maneuver (shown in the Jupiter-centered inertial frame), and (c) getting captured by Europa (shown in the Jupiter-Europa rotating frame).

We have quite a bit of freedom in choosing initial velocities. Within certain ranges of $(\dot{x}, \dot{y})$, a wide variety of "capture"-like behavior is possible. We choose trajectories which wind around $L_{1}$ or $L_{2}$ orbits, because these trajectories are known to be more versatile in mission design.

One might expect that dynamical channels of one moon would be so perturbed by the presence of the other moon, that their "intersection" would disappear and no transfer could be found. But, as we show, this is not the case. Because the intersection occurs far from both moons, in a jovicentric region of phase space, we expect that the effect of one moon on the other moon's dynamical channel would be negligible. Also, the mass parameters of the moons involved, rough measures of their gravitational perturbation, are quite small $\left(\approx 10^{-5}\right)$.

Modifications to the trajectory are necessary to fine tune the behavior near each moon. The Ganymede $L_{1}$ Lyapunov orbit is so large that the backward integrated trajectory passes by Ganymede without any revolutions about the moon. A $\Delta V$ of $157 \mathrm{~m} / \mathrm{s}$, performed near Ganymede, is necessary to add one full loop around the moon. Similarly, to effect a near circular orbit once the forward integrated trajectory is temporarily captured by Europa, a $\Delta V$ of $87 \mathrm{~m} / \mathrm{s}$, performed at closest approach to Europa, is necessary. Thus, the total $\Delta V$ for the trajectory shown in Figure 3.3 is $1452 \mathrm{~m} / \mathrm{s}$, still substantially lower than the Hohmann transfer value.

Using the trajectory from the bi-circular model, an end-to-end trajectory can be computed in the JPL ephemeris model using JPL's LTool (Libration Point Mission Design Tool) currently under development. This is the subject of a future paper.

\section{Conclusions and Future Work.}

This paper used dynamical systems techniques to investigate transport channels in the phase space which could be exploited by spacecraft to "leap-frog" from moon to moon within a planet's satellite system using low-fuel controls. This method of space mission design is an elaboration upon the patched conic (2-body) approach, toward a patched 3-body approach.

We can conduct a "Petit Grand Tour" of the Jovian moon system by applying the coupled 3-body 
model. The delicate dynamics of the 4-body problem are well approximated by the coupled 3-body problem for a satellite system such as the Galilean moons of Jupiter. By systematically seeking heteroclinic connections between Lagrange point orbits of adjacent moons, one can design trajectories which transfer from the vicinity of one moon to another using little fuel. One can seek intersections between the channels of transit orbits enclosed by the stable and unstable manifold tubes of Lyapunov orbits of different moons using the method of Poincaré section. With maneuvers sizes $(\Delta V)$ much smaller than that necessary for Hohmann transfers, transfers between moons are possible.

Methods such as those developed here can be applied to other 4-body systems, such as the Sun-EarthMoon-spacecraft system. We can produce a low-fuel transfer to the Moon, like the 1991 Hiten mission which effected a ballistic capture at the Moon with less propulsion than a standard Hohmann transfer (Belbruno and Miller [1993]). We refer to this mission concept as "Shoot the Moon." See Figure 4.1. Details of the mission design procedure appear in Koon, Lo, Marsden, and Ross [2000b].

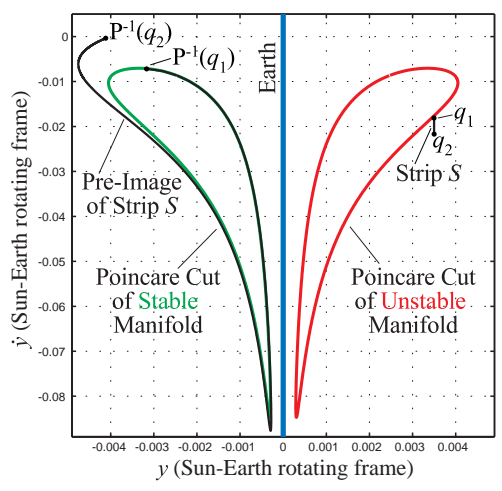

(a)

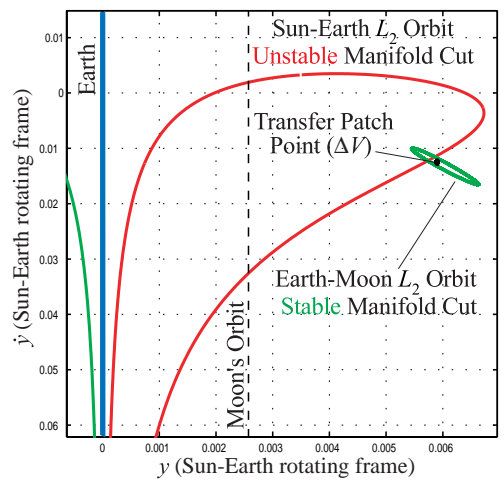

(b)

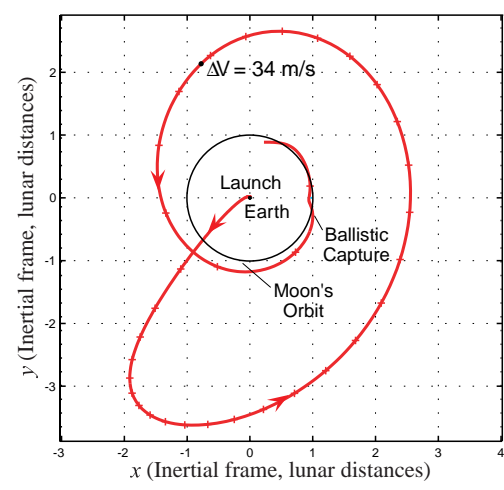

(c)

Figure 4.1: (a) Behavior of orbits near and outside the stable and unstable manifolds of the Sun-Earth $L_{2}$ orbit in the Earth region. (b) Intersection between Sun-Earth $L_{2}$ orbit unstable manifold and Earth-Moon $L_{2}$ orbit stable manifold (small oval). (c) The Earth to Moon transfer with ballistic capture by the Moon.

Systematic Approach to Coupled Three-Body Problem. We have shown an example where the coupled 3-body problem worked well as an approximation to 4-body dynamics. The next step is to develop a theoretical understanding for when the coupled 3-body problem is an accurate approximation to larger $N$-body dynamics, and to what degree. For instance, it is desirable to know what degree of distortion a fourth massive body has on the invariant manifold structures of the Lagrange point orbits, particularly the tubes containing transit orbits. The effect is likely to be a complicated interplay of the relative masses of the bodies, their relative orbital distances, and the relative positions of the bodies during the time intervals of interest. A firmer foundation for understanding these and other effects will allow one to produce further optimized trajectories.

Extension to Three Dimensions. We plan to extend our techniques to the 3-dimensional CR3BP and coupled CR3BP by seeking homoclinic and heteroclinic orbits associated with 3-dimensional periodic "halo" and quasi-periodic "quasi-halo" and Lissajous orbits about $L_{1}$ and $L_{2}$. Their union is a set of 3-dimensional homoclinic-heteroclinic chains around which symbolic dynamics could catelog a variety of exotic orbits. Dynamical channels in the form of codimension-1 surfaces in the energy surface likely exist (analogous to the tubes in the planar problem) which separate qualitatively different types of motion, such as transit and non-transit orbits.

Merging Optimal Control with Dynamical Systems Theory. The construction of exotic spacecraft orbits using patched 3-body dynamical channels requires optimal, fuel minimizing impulsive and 
continuous propulsion thruster controls to navigate these dynamically sensitive regions of phase space. A deeper understanding of the dynamical structure of the restricted 3-body problem may suggest alternative formulations of the optimizing scheme which are based more on the geometry of the phase space. Algorithms could be developed with the natural dynamics built in, thereby yielding better convergence properties. It would also be interesting to explore additional ways to use optimal control in the presence of mechanics (as in, for example, Koon and Marsden [1997]).

Acknowledgments. We thank Gerard Gómez and Josep Masdemont for sharing their wonderful software tools. The name "Petit Grand Tour" was suggested by Nelson Carter, JPL Documentation Services.

This work was carried out at the Jet Propulsion Laboratory and the California Institute of Technology under a contract with the National Aeronautics and Space Administration. In addition, the work was partially supported by the Caltech President's fund, the NASA Advanced Concepts Research Program, The Ltool Project, and NSF grant KDI/ATM-9873133.

\section{References}

Abraham, R. and J.E. Marsden [1978] Foundations of Mechanics. Second Edition, Addison-Wesley.

Conley, C. [1963] On some new long periodic solutions of the plane restricted 3-body problem. Comm. Pure Appl. Math. 16, 449-467.

Belbruno, E and J. Miller [1993] Sun-perturbed earth-to-moon transfers with ballistic capture, Journal of Guidance, Control, and Dynamics 16, 770-775.

Conley, C. [1968] Low energy transit orbits in the restricted 3-body problem. SIAM J. Appl. Math. 16, $732-746$.

Gómez, G., A. Jorba, J. Masdemont, and C. Simó [1991] Study Refinement of Semi-Analytical Halo Orbit Theory, Final Report, ESOC Contract No.: 8625/89/D/MD(SC), Barcelona, April, 1991.

Howell, C., B. Barden and M. Lo [1997] Application of dynamical systems theory to trajectory design for a libration point mission, The Journal of the Astronautical Sciences 45(2), April 1997, 161-178.

Koon, W.S. and J.E. Marsden [1997] Optimal control for holonomic and nonholonomic mechanical systems with symmetry and Lagrangian reduction. SIAM J. Control and Optim. 35, 901-929.

Koon, W.S., M.W. Lo, J.E. Marsden and S.D. Ross [2000a] Heteroclinic connections between periodic orbits and resonance transitions in celestial mechanics. Chaos 10(2), June 2000, 427-469.

Koon, W.S., M.W. Lo, J.E. Marsden and S.D. Ross [2000b] Shoot the Moon, AAS/AIAA Astrodynamics Specialist Conference, Florida, 2000, AAS 00-166.

Llibre, J., R. Martinez and C. Simó [1985] Transversality of the invariant manifolds associated to the Lyapunov family of perodic orbits near L2 in the restricted 3-body problem, Journal of Differential Equations 58, 104-156.

Lo, M. and S. Ross [1997] SURFing the Solar System: Invariant Manifolds and the Dynamics of the Solar System JPL IOM 312/97, 2-4.

Lo, M. and S. Ross [1998] Low energy interplanetary transfers using invariant manifolds of L1, L2 and halo orbits, AAS/AIAA Space Flight Mechanics Meeting, Monterey, California, 9-11 February 1998.

Lo, M., B.G. Williams, W.E. Bollman, D. Han, Y. Hahn, J.L. Bell, E.A. Hirst, R.A. Corwin, P.E. Hong, K.C. Howell, B. Barden, and R. Wilson [1998] Genesis Mission Design, Paper No. AIAA 98-4468.

Marsden, J.E. and T.S. Ratiu [1999] Introduction to Mechanics and Symmetry. Texts in Applied Mathematics, 17, Springer-Verlag, 1994. Second Edition, 1999.

McGehee, R. P. [1969] Some homoclinic orbits for the restricted 3-body problem, Ph.D. thesis, University of Wisconsin.

Meyer, K.R. and R. Hall [1992] Hamiltonian Mechanics and the n-body Problem. Springer-Verlag, Applied Mathematical Sciences.

Szebehely, V. [1967] Theory of Orbits. Academic Press, New York/London.

Serban, R., W.S. Koon, M.W. Lo, J.E. Marsden, L.R. Petzold, S.D. Ross and R.S. Wilson [2000] Optimal Control for Halo Orbit Missions, submitted to Automatica. 